\title{
Synthesis of Some New Thioethers and 4-Thiazolidinones Bearing 3-(Pyridine-4'-yl)-1,2,4-Triazino[5,6-b]Indole Moiety as Antifungal Agents
}

\author{
Wafa A. Baker Bawazir \\ Department of Chemistry, Faculty of Science, KAU, Jeddah, Saudi Arabia \\ Email: wbawazeer@kau.edu.sa
}

How to cite this paper: Bawazir, W.A.B. (2019) Synthesis of Some New Thioethers and 4-Thiazolidinones Bearing 3-(Pyridine4'-yl)-1,2,4-Triazino[5,6-b] Indole Moiety as Antifungal Agents. International Journal of Organic Chemistry, 9, 37-46.

https://doi.org/10.4236/ijoc.2019.91004

Received: February 15, 2019

Accepted: March 8, 2019

Published: March 11, 2019

Copyright $\odot 2019$ by author(s) and Scientific Research Publishing Inc. This work is licensed under the Creative Commons Attribution International License (CC BY 4.0).

http://creativecommons.org/licenses/by/4.0/

\begin{abstract}
Some new asymmetric thioethers 5 and 4-thiazolidinones 6 have been obtained from condensation of 5-formyl-3-(pyridin-4'-yl)-1,2,4-triazino[5,6- $b$ ] indole (3) with halogenated aromatic amines followed by addition of thiophenol and/or cycloaddition with thiolactic acids in nonpolar solvents. Structures of the products confirmed by elemental analysis and spectral measurements. The new systems obtained were evaluated as antifungal agents.
\end{abstract}

\section{Keywords}

Thioethers, Thiazolidnones, 1,2,4-Triazino Indole, Fungicidal, Cycloaddition

\section{Introduction}

Heterocyclic systems of 1,2,4-Triazino[5,6-b] indole have been successfully used as a carrier for diverse functional groups in the development of some antiviral agents [1]. Various studies found the efficacy of such nucleus in the production of antibacterial [2], antifungal [3], inhibitors of blood platelet aggregation, anti-hypertensive [4] agents. As well as 1,2,4-triazino[5,6- $b]$ indoles derivatives apply useful applications in medicinal chemistry, especially, antimalarial [5], and antidepressant agents [6]. Beside, 3,5-disubstituted-1,2,4-triazino[5,6- $b$ ]indoles exhibited an important biological activity as antileishmanial [7], antihypoxic [8] and anti-inflammatory activities [9]. On the other hand, recently found thiazolidinones showed a high activity towards the most fungi [10]. Based upon these observations, and in search for obtain a newly systems combined between thioether bearing 1,2,4-triazino[5,6- $b$ ]indole and thiazolidinones [11] [12]. Asym- 
metrical thioether bearing 1,2,4-triazine moiety exhibited anticancer and anti-HIV activity [10].

The present work tends to synthesize some new thiazolidinones bearing a triazinoindolein view of their effects and fungicidal activity.

\section{Chemistry}

A novel route to synthesize 3,5-disubstituted heterocyclic-1,2,4-triazino[5,6- $b$ ] indoles deduced from condensation of isatin with isonicotinic acid hydrazidevia reflux in $\mathrm{MeOH}$ - drops $\mathrm{AcOH}$ to give 3-hydrazono-indol-2(1H)one (1) which upon fused with ammonia acetate in glacial $\mathrm{AcOH}$, yielded 3-(pyridin-4'-yl)-5 H1,2,4-triazino[5,6- $b]$ indole (Scheme 1). Careful formylation of compound 2 via stirring with formyl acetate (prepared by reflux equimolar amounts of $\mathrm{HCO}_{2} \mathrm{H}$ with $\mathrm{Ac}_{2} \mathrm{O}$ ) in dry ether at room temperature, afforded 3-(pyridin-4'-yl)-5-formyl-1,2,4-triazino[5,6-b] indole (3) (Scheme 1). Compound 3 underwent condensation with primary aromatic amines in EtOH yielded the Schiffbases 4 (Scheme 2). Simple 1,2-addition to $-\mathrm{CH}=\mathrm{N}$-Ar of compounds $4 \mathrm{a}$-cunder reflux with thiophenol in ethylbenzene to give the thioethers 5a-c (Scheme 2).

As well as cycloaddition of thiolactic acid to $-\mathrm{CH}=\mathrm{N}$-Arof compounds4a-c, via reflux in dioxan, led to the direct formation of 4-thiazolidinones $6 \mathrm{a}-\mathrm{c}$, respectively, (Figure 1).

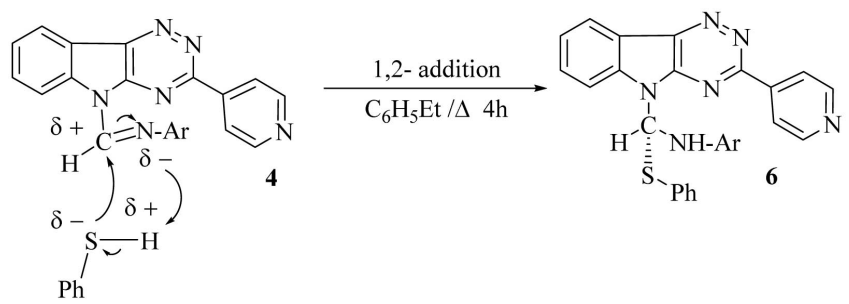

Figure 1. A plausible mechanism of 1,2-addition reaction of 4 to produce 6 .
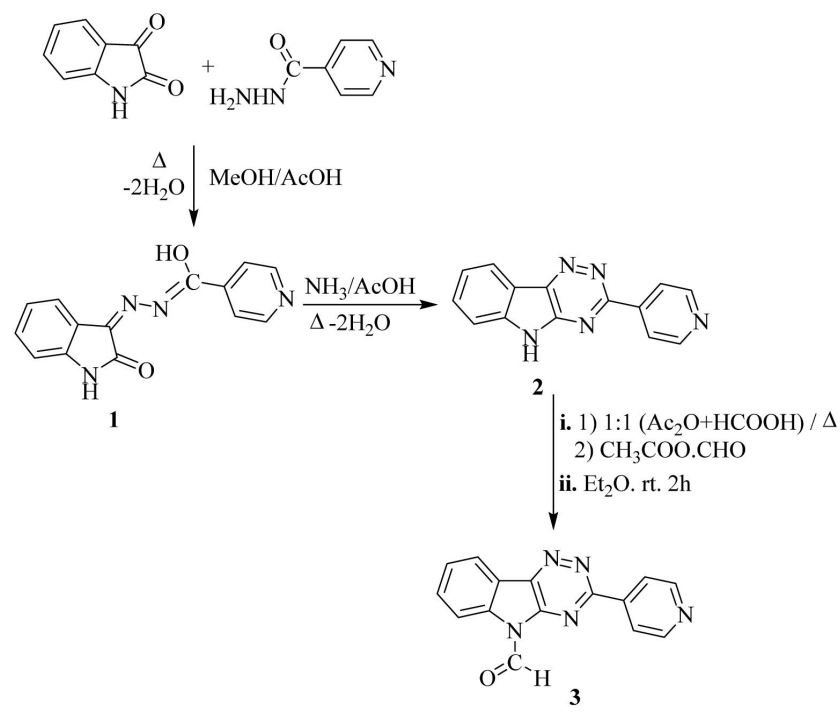

Scheme 1. Synthesis of compound 3. 


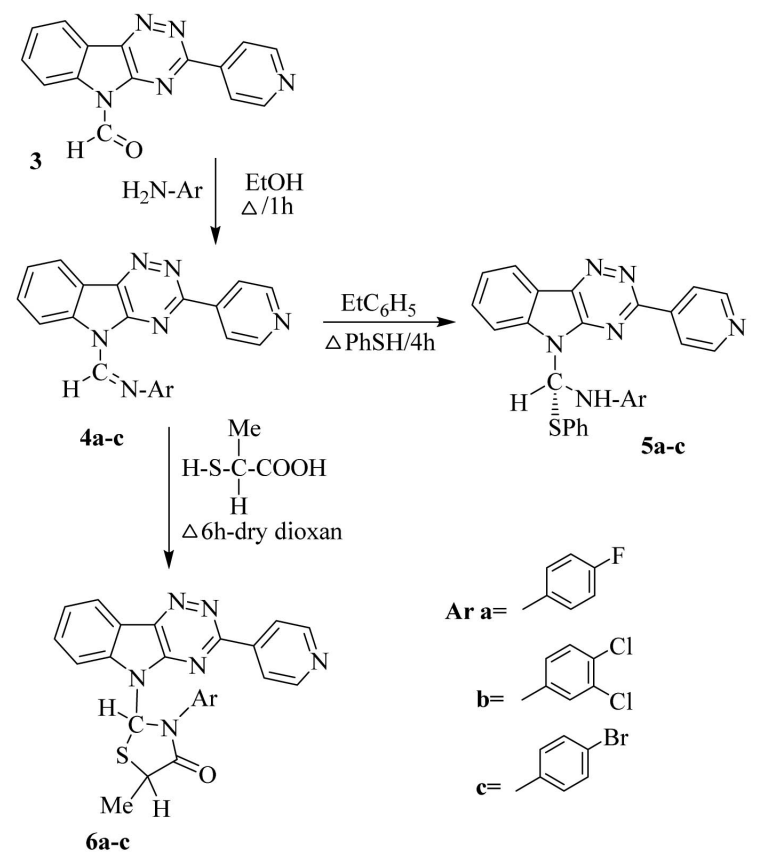

Scheme 2. Formation of compounds 5 \& 6 from 4 .

\section{Results and Discussion}

Former structure of the synthesized compounds deduced from their correct elemental analysis and spectral data. IR spectrum of compound 1 showed $v$ at $3400-3300$ and $3190 \mathrm{~cm}^{-1}$ for $\mathrm{OH}$ and $\mathrm{NH}$, while lacking of $\mathrm{OH}$ and $\mathrm{C}=\mathrm{O}$ groups for 2. Compound 3 showed the presence of $v$ at $1738 \mathrm{~cm}^{-1}$ for $\mathrm{CHO}$ formed and absence NH. IR spectra of compounds 4 and 6 recorded a lack of $\mathrm{NH}$ functional group.

On the other hand, IR spectra of 5 and 6 showed $v$ at $1200-1180 \mathrm{~cm}^{-1}$ for C-S-C and $v$ at $1700-1680 \mathrm{~cm}^{-1}$ for $\mathrm{C}=\mathrm{O}$ of compounds 6 with $v$ at 2900,2850 , 1480 and $1440 \mathrm{~cm}^{-1}$ attribute to aliphatic groups. All the new fluorinated compounds obtained showed $v$ at $1250-1240 \mathrm{~cm}^{-1}$ for C-F, and $v$ at $1620-1590$ $\mathrm{cm}^{-1}$ for cyclic $\mathrm{C}=\mathrm{N}$ groups of pyridine and 1,2,4-triazine.

${ }^{1}$ HNMR spectra of the compounds 2-6 give us good indications about their line structures. Only compound 3 showed a resonated signal at $8.80 \mathrm{ppm}$ for formyl proton, while that of compounds 2 and 5 recorded $\delta$ at $11.43 \mathrm{ppm}$ attribute to NH protons. ${ }^{1} \mathrm{HNMR}$ spectra of compounds $\mathbf{4 , 5}$ and $\mathbf{6}$ showed $\delta$ at 8.5, $7.8-7.7$ and $2.52 \mathrm{ppm}$ for pyridine, $\mathrm{CH}=\mathrm{N}$ and $\mathrm{CH}_{3}$ protons. All the 4-thiazolidinone derivatives $6 \mathrm{a}-\mathrm{c}$ showed a resonated signal at $\delta 3.53 \mathrm{ppm}$ attribute to $\mathrm{C} 5-\mathrm{H}$.

${ }^{13} \mathrm{CNMR}$ spectra of the compounds 3-6 showed $\delta$ at 162, 155, 145, 140, 130 120 and $77-44 \mathrm{ppm}$ attribute to $\mathrm{C}=\mathrm{O}, \mathrm{C}-\mathrm{S}-\mathrm{C}, \mathrm{C}-\mathrm{F}, \mathrm{C}=\mathrm{N}$, pyridine aromatic and aliphatic carbons.

In addition, mass spectrometric study of some compounds recorded the molecular ion peak at lower integration which upon further fragmentation process give a base peak at lower integration (Figure 1 and Figure 2). For example, $\mathrm{M} / z$ 


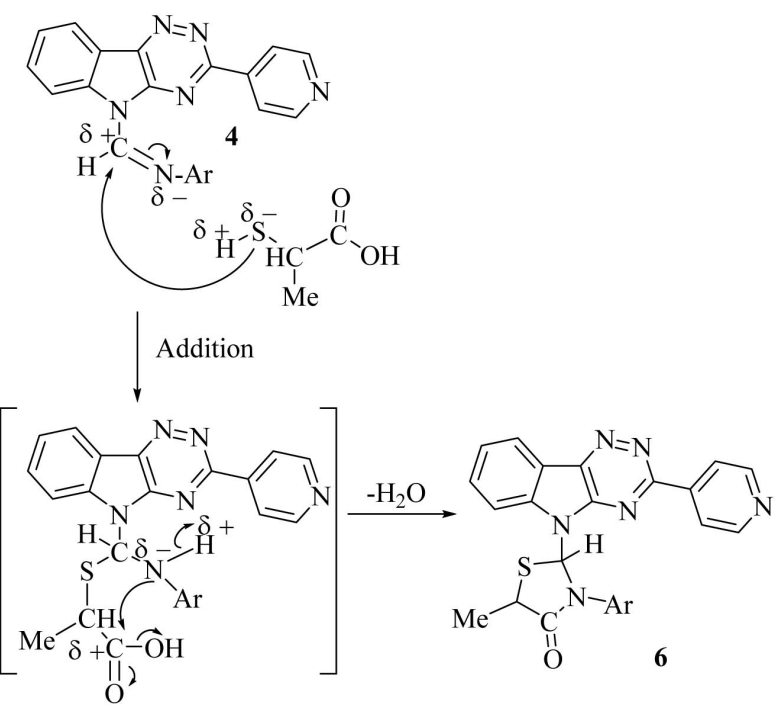

Figure 2. Formation of compound 6 from 4.

of compound 3 recorded a base peak at 116 attribute to the indolyl ion, while that of $\mathbf{6 a}$ exhibited a base peak at 60 . Stability of base peak for compound 6 may be due to a type of hyperconjugation bonded to thiirene radical, a plausible Mass fragmentation patterns of compounds 3 and $6 \mathrm{a}$ are illustrated in (Figure 3 and Figure 4) respectively.

\section{Antifungal Activity}

4-Thiazolidinone derivatives exhibited a wide range of biological activity especially as antifungal agents [11] [13], also, thioethers bearing a heterocyclic nitrogen systems used as anticancer, and anti-HIV probes [10]. Besides, the 1,2,4-triazino[5.6- $b$ ] indole, showed various biological activities [4] [5] [6] [7]. Based on these observations, the present work tends to synthesize some new systems combined between these biocidal agents. The new compounds containing $\mathrm{S}, \mathrm{F}, \mathrm{Cl}$ and $\mathrm{Br}$ atoms (5 - 6) were evaluated as antifungal agents against $A s$ pergillus niger and Alternaria alternata fungi, using the diffusion method [14] [15].

The tested compounds dissolved in DMF $\left(1 \mathrm{mg} \cdot \mathrm{ml}^{-1}\right)$ and the antibiotic Fluconazole used as a standard drug. The inhibition zones (microbial growth surrounding the filter paper disc $(2.5 \mathrm{~mm})$ were measured at the incubation period at $30^{\circ} \mathrm{C}$ for 3 days. The inhibition zones of the organisms measured by a zone surrounding each disk and reported in Table 1.

From the results obtained from the Table 1 we can conclude that:

1) The compounds containing $\mathrm{F}$ atoms are more active than that contain $\mathrm{Cl}$ and $\mathrm{Br}$.

2) The more sulfur element percentage, showed higher inhibition zones towards fungi.

3) The compounds contains both F and S are very active than other compounds. 


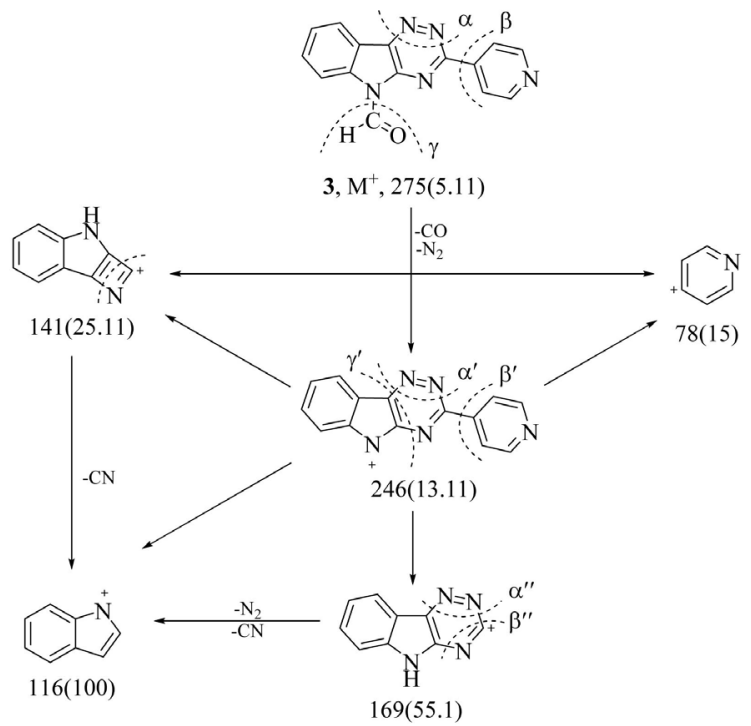

Figure 3. A suggested Mass fragmentation pattern of compound 3.

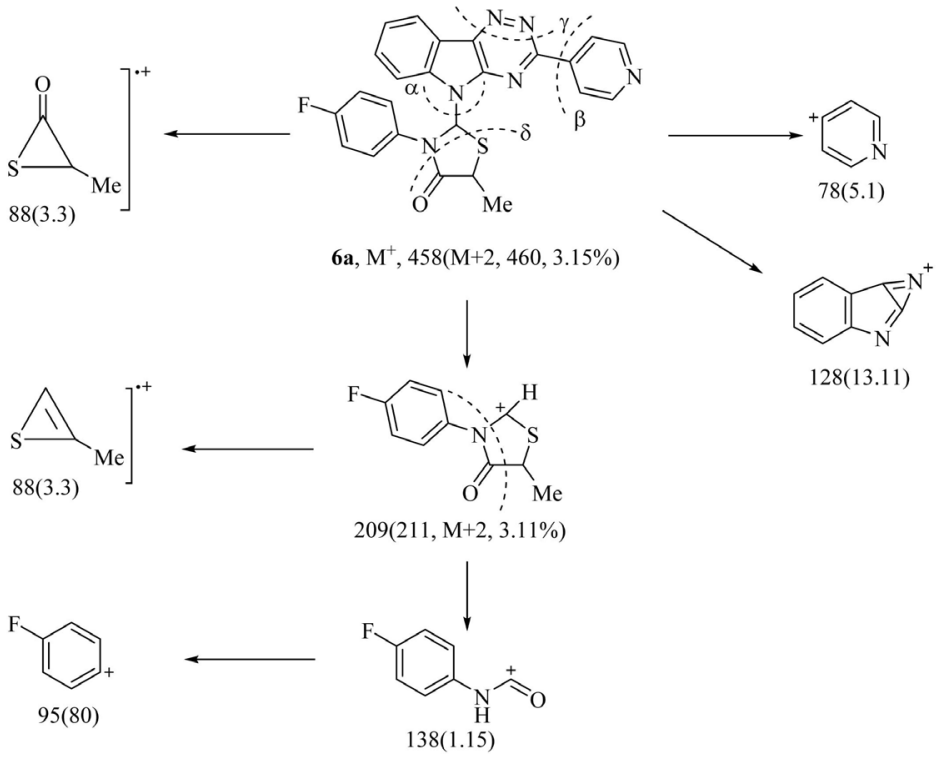

Figure 4. Mass fragmentation pattern of compound 6a.

Table 1. The fungicidal effects of some synthesized compounds.

\begin{tabular}{cccc}
\hline \multirow{2}{*}{ Compounds } & S\% & \multicolumn{2}{c}{ Inhibition Zone $\left(\mathrm{IZ} \mathrm{mg \cdot \textrm {mL } ^ { - 1 } )}\right.$} \\
\cline { 3 - 4 } & & Aspergillus $N$ & Alternaria $A$. \\
\hline 5a & 6.61 & 29 & 26 \\
5b & 6.04 & 27 & 26 \\
5c & 5.93 & 25 & 25 \\
6a & 6.98 & 30 & 27 \\
6b & 6.28 & 28 & 26 \\
6c & 6.16 & 28 & 25 \\
Fluconazole & - & 25 & 25
\end{tabular}


4) The antifungal activities are in the order: $6 a>5 a>6 b, 6 c>5 b>5 c$ for Aspergillus $N$. and $6 \mathrm{a}>5 \mathrm{a}, 5 \mathrm{~b}, 6 \mathrm{~b}>5 \mathrm{c}, 6 \mathrm{c}$ for Alternaria $A$. fungi.

5) Most of the evaluated compounds showed IZ more than that of Fluconazole which makes these fluorine substituted sulfur systems good fungicidal probes.

\section{Experimental}

Melting points determined with an electrothermal Bibby Stuart Scientific melting point sample (UK). A Perkin Elmer Model PXI-FT system 55,529 used for recording IR spectra of the prepared compounds. A Bruker advance DPX 400 $\mathrm{MHz}$ model uses TMS as an internal standardwas used for recording the ${ }^{1} \mathrm{H}$ and ${ }^{13} \mathrm{CNMR}$ spectra of the compounds on deuterated DMSO- $d_{6}$. A GC-MS-GP 1000 Ex model used for recording the mass spectra of the compounds. Electronic spectra recorded in ethanol on Shimadzu UV and visible 310 IPC Spectrophotometer.Elemental analysis performed in the microanalytical center of Cairo University, Cairo, Egypt. Biological activity carried out in Biology center, Faculty of Science, Ain Shams University, Egypt.

\section{- N-(2-Oxoindolin-3-ylidene)isonicotinohydrazonic acid(1)}

Equimolar mixture of isatin and isonicotinic acid hydrazide in $\mathrm{MeOH}$ (100 $\mathrm{ml}$ ) with drops of $\mathrm{AcOH}$, heated underreflux for 1h, cooled. The solid obtained filtered off and crystallized from $\mathrm{MeOH}$ to give 1 as orange crystals. Yield 78\%, M.p: $289^{\circ} \mathrm{C}-290^{\circ} \mathrm{C}$. IR (v) cm ${ }^{-1}: 3400-3300$ (b, OH), $3190(\mathrm{NH}), 1697$ (C=O), 1620, $1604(\mathrm{C}=\mathrm{N}), 1300(\mathrm{~N}-\mathrm{N}), 862.6,811$ (aromatic CH). ${ }^{1} \mathrm{H}-\mathrm{NMR}$ (DMSO- $d_{6}$ ) $\delta$ ppm: $13.99(s, 1 \mathrm{H}, \mathrm{NH}), 10.81(s, 1 \mathrm{H}, \mathrm{OH}), 8.88-8.81,8.00-8.04(d, d, 2 \mathrm{H}$, of pyridine), 7.91 - 7.6 ( $m, 2 \mathrm{H}$, pyridine), $7.44-7.07$ ( $m, 4 \mathrm{H}$, aromatic protons). ${ }^{13} \mathrm{CNMR}$ (DMSO- $\left.d_{6}\right) \delta$ ppm: $162(\mathrm{C}=\mathrm{O} \rightleftarrows \mathrm{C}-\mathrm{OH}), 142(\mathrm{C}=\mathrm{N}), 132.21-121.20$ (aromatic carbons), 119.54, $111.32(\mathrm{C}-\mathrm{N})$. Anal. Calcd: C, 63.5; H, 3.75; N, $21.05 \%$ for $\mathrm{C}_{14} \mathrm{H}_{10} \mathrm{~N}_{4} \mathrm{O}_{2}$ (266). Found: C, 63.21; H, 7.59; N, 20.85\%.

\section{- 3-(Pyridin-4'-yl)-5H-1,2,4-triazino[5,6-b]indole (2)}

A mixture of $1(5 \mathrm{~g})$ and ammonium acetate $(5 \mathrm{~g})$ in glacial acetic acid $(10 \mathrm{ml})$ heated underreflux for $2 \mathrm{~h}$, cooled then washed with cold water and crystallized from $\mathrm{EtOH}$ to give 2. Yield 65\%. M.p: $296^{\circ} \mathrm{C}-297^{\circ} \mathrm{C}$. IR (v) $\mathrm{cm}^{-1}: 3191(\mathrm{NH})$, 1624, 1619, $1597(\mathrm{C}=\mathrm{N}), 1341(\mathrm{NCN}), 893,846$ (aromatic $\mathrm{CH}) .{ }^{1} \mathrm{HNMR}$ (DMSO- $\left.d_{6}\right) \delta$ ppm: $13.98(s, 1 \mathrm{H}, \mathrm{NH}), 8.8-8.86(d, d, 2 \mathrm{H}$, pyridine), $7.78-7.03$ ( $m$, $4 \mathrm{H}$, aromatic protons). ${ }^{13} \mathrm{CNMR}$ (DMSO- $d_{6}$ ) $\delta$ ppm: $149.9,142.72,139.2$ $(\mathrm{C}=\mathrm{N}), 132.20(\mathrm{C}-\mathrm{N}), 122.84-121.20$ (aromatic carbons), 119.54, 111.32 (C5 C6 of 1,2,4-triazine). Anal. Calcd: C, 68.01; H, 3.64; N, 28.34\% for $\mathrm{C}_{14} \mathrm{H}_{9} \mathrm{~N}_{5}(247)$. Found: C, $67.85 ; \mathrm{H}, 3.55 ; \mathrm{N}, 28.11 \%$.

\section{- 3-(Pyridin-4'-yl)-5-formyl-1,2,4-triazino[5,6-b]indole (3)}

A mixture of $2(0.01 \mathrm{~mol})$ and formyl acetate $(0.01 \mathrm{~mol}$ prepared by reflux of $10 \mathrm{ml} \mathrm{Ac}_{2} \mathrm{O}$ with $10 \mathrm{ml} \mathrm{HCOOH}$ for $\left.10 \mathrm{~min}\right)$ in diethyl ether $(100 \mathrm{ml})$ then stir along $2 \mathrm{~h}$. The solid produced filtered off and crystallized from dioxane to give 3 . Yield 60\%, M.p: $304^{\circ} \mathrm{C}-305^{\circ} \mathrm{C}$. IR (v) $\mathrm{cm}^{-1}: 3030$ (aromatic $\mathrm{CH}$ ), $1738(\mathrm{C}=\mathrm{O})$, $1589(\mathrm{C}=\mathrm{N}), 880,820$ (aromatic CH). ${ }^{1} \mathrm{HNMR}\left(\mathrm{DMSO}-d_{6}\right) \delta \mathrm{ppm:} 9.2(s, 1 \mathrm{H}$, 
$\mathrm{CHO}), 8.88,8.82(d, d, 2 \mathrm{H}$, pyridine), $8.01-7.80$ (2H, pyridine), $7.61-6.92(\mathrm{~m}$, $4 \mathrm{H}$, aromatic protons); ${ }^{13} \mathrm{CNMR}\left(\mathrm{DMSO}-d_{6}\right) \delta \mathrm{ppm}: 164.49(\mathrm{C}=\mathrm{O}), 150.86$, $150.26,144.20,142.73(\mathrm{C}=\mathrm{N}), 140.10,139.21(\mathrm{C}-\mathrm{N}), 127.11$ - 121.20 (aromatic carbons); 119.55, 115.46 (C5-C6 of 1,2,4-triazine). Anal. Calcd: C, 65.45; H, 3.27; $\mathrm{N}, 25.45 \%$ for $\mathrm{C}_{15} \mathrm{H}_{9} \mathrm{~N}_{5} \mathrm{O}$ (275). Found: C, 65.15; H, 3.12; N, 25.30\%.

\section{- Schiff's base (4a-c)}

A mixture of $3(0.01 \mathrm{~mol})$ and primary aromatic amines, such as 4 -fluoroaniline, 3,4-dichloroaniline and/or 3-bromoaniline $(0.01 \mathrm{~mol})$ were heated in in EtOH $(100 \mathrm{ml})$ under reflux for $1 \mathrm{~h}$, cooled. The resulted solid filtered off and crystallized from $\mathrm{EtOH}$ to produce compounds $4 \mathrm{a}-\mathrm{c}$.

4a: Yield 60\%, M.p: $297^{\circ} \mathrm{C}-298^{\circ} \mathrm{C}$. IR (v) $\mathrm{cm}^{-1}: 2941,2864$ (aliphatic CH), $1629(\mathrm{C}=\mathrm{N}), 1364(\mathrm{NCN}), 1255(\mathrm{C}-\mathrm{F}), 912,865,802$ (aromatic CH), 765 (C-F). ${ }^{1} \mathrm{HNMR}\left(\mathrm{DMSO}-d_{6}\right) \delta$ ppm: $8.95(s, 1 \mathrm{H}, \mathrm{CH}=\mathrm{N}), 8.88-8.87(d, d, 2 \mathrm{H}$, pyridine), $7.81-7.62\left(m, 2 \mathrm{H}\right.$, pyridine). ${ }^{13} \mathrm{CNMR}$ (DMSO- $\left.d_{6}\right) \delta \mathrm{ppm}: 150.8(\mathrm{CH}=\mathrm{N})$, 142.74, $139.23(\mathrm{C}=\mathrm{N}), 132.21(\mathrm{C}-\mathrm{N}), 122.86$ - 119.56 (aromatic C-C). Anal. Calcd: C, 68.47; H, 3.53; N, 22.8; F, 5.16\% for $\mathrm{C}_{21} \mathrm{H}_{13} \mathrm{~N}_{6} \mathrm{~F}$ (368). Found: C, 68.31; $\mathrm{H}, 3.25 ; \mathrm{N}, 22.55 ; \mathrm{F}, 5.01 \%$.

4b: Yield 72\%, M.p: $293^{\circ} \mathrm{C}-295^{\circ} \mathrm{C}$. IR ( v) $\mathrm{cm}^{-1}: 3050$ (aromatic CH), 2917, 2880(aliphatic CH), $1625(\mathrm{C}=\mathrm{N}), 1479$ (aliphatic $\mathrm{CH}), 1364(\mathrm{NCN}), 910,864$ (aromatic CH), 675,647 (C-Cl). Anal. Calcd: C, 60.14; H, 2.86; N, 20.04; $\mathrm{Cl}_{2}$, $16.94 \%$ for $\mathrm{C}_{21} \mathrm{H}_{12} \mathrm{~N}_{6} \mathrm{Cl}_{2}$ (419). Found: C, 59.85; H, 2.69; N, 19.84; F, $16.79 \%$.

4c: Yield 55\%, M.p: $182^{\circ} \mathrm{C}-185^{\circ} \mathrm{C}$. IR $(v) \mathrm{cm}^{-1}$ : 3050 (aromatic CH), 2980 (aliphatic $\mathrm{CH}), 1625(\mathrm{C}=\mathrm{N}), 1488$ (aliphatic $\mathrm{CH}), 1364(\mathrm{NCN}), 910,864,804$ (aromatic CH), 700 (C-Br). Anal. Calcd: C, 58.74; H, 3.03; N, 19.58; Br, 18.6\% for $\mathrm{C}_{21} \mathrm{H}_{13} \mathrm{~N}_{6} \mathrm{Br}$ (429). Found: C, 58.59; H, 2.89; N, 19.40; $\mathrm{Br}, 18.49 \%$.

\section{- Asymmetric thioethers (5a-c)}

Compounds $4 \mathrm{a}-\mathrm{c}(0.5 \mathrm{~g})$ and thiophenol $(3 \mathrm{ml})$ in ethyl benzene $(100 \mathrm{ml})$ heated under reflux for $4-6 \mathrm{~h}$, cooled then added petroleum ether $\left(40^{\circ} \mathrm{C}-60^{\circ} \mathrm{C}\right.$, $100 \mathrm{ml}$ ). The solid thus obtained filtered off and crystallized from dioxane to give $\mathbf{5 a - c}$ respectively.

5a: Yield 62\%, M.p: $78^{\circ} \mathrm{C}-80^{\circ} \mathrm{C}$. IR (v) $\mathrm{cm}^{-1}: 3149$ (aromatic CH), 2920, 2880 (aliphatic CH), $1618(\mathrm{C}=\mathrm{N}), 1480$ (aliphatic CH), 1234 (C-F), 1099 (C-S-C), 890, 867 (aromatic CH), 788 (C-F). ${ }^{1} \mathrm{HNMR}$ (DMSO- $\left.d_{6}\right) \delta$ ppm: $11.32(s, 1 \mathrm{H}, \mathrm{NH})$, 7.62 - $7.60(s, 1 \mathrm{H}, \mathrm{CH}-\mathrm{N}), 7.405,7.43(d, d, 2 \mathrm{H}$, pyridine), 7.417, $7.44(d, d, 2 \mathrm{H}$, adjacent C-F), 7.29, 7.27 ( $m, 2 \mathrm{H}$, pyridine), 7.226 - 7.208, 7.177 - 6.984 (each $m$, $6 \mathrm{H}$, aromatic protons). ${ }^{13} \mathrm{CNMR}$ (DMSO- $\left.d_{6}\right) \delta \mathrm{ppm}: 175$ (C-S), 162.61 (S-C-N), $143.71(\mathrm{C}-\mathrm{F}), 132.13(\mathrm{C}=\mathrm{N}), 128.25$ - 122.71 (aromatic carbons), 119.11, 111.38 (C5 - C6 of 1,2,4-triazine). Anal. Calcd: C, 67.78; H, 3.97; N, 17.57; F, 3.97; S, $6.69 \%$ for $\mathrm{C}_{27} \mathrm{H}_{19} \mathrm{~N}_{6} \mathrm{FS}$ (478). Found: C, 67.59; H, 3.85; N, 17.33; F, 3.79; S, 6.55\%.

5b: Yield 75\%, M.p: $59^{\circ} \mathrm{C}-60^{\circ} \mathrm{C}$. IR $(v) \mathrm{cm}^{-1}: 3085$ (aromatic $\mathrm{CH}$ ), 2950, 2885 (aliphatic $\mathrm{CH}), 1710(\mathrm{C}=\mathrm{O}), 1610(\mathrm{C}=\mathrm{N}), 1488$ (deformation $\left.\mathrm{CH}_{3}\right), 1080$ (C-S-C), 910, 880, 810 (aromatic CH), 700 (C-Cl). Anal. Calcd. C, 61.24; H, 3.40; $\mathrm{N}, 15.90 ; \mathrm{Cl}, 13.42 ; \mathrm{S}, 6.04 \%$ for $\mathrm{C}_{27} \mathrm{H}_{18} \mathrm{~N}_{6} \mathrm{Cl}_{2} \mathrm{~S}$ (529). Found C, 61.18; H, 3.25; N, $15.81 ; \mathrm{S}, 5.89 ; \mathrm{Cl}, 13.38 \%$. 
5c: Yield 58\%, M.p: $68^{\circ} \mathrm{C}-70^{\circ} \mathrm{C}$. IR (v) $\mathrm{cm}^{-1}: 3065$ (aromatic CH), 2910, 2880 (aliphatic $\mathrm{CH}$ ), $1700(\mathrm{C}=\mathrm{O}), 1610(\mathrm{C}=\mathrm{N}), 1488,1444$ (deformation of $\left.\mathrm{CH}_{3}\right), 1090$ (C-S-C), 915, 880, 820 (aromatic CH), 680 (C-Br). Anal. Calcd: C, 60.11; H, 3.52; $\mathrm{N}, 15.58 ; \mathrm{Br}, 14.84 ; \mathrm{S}, 5.93 \%$ for $\mathrm{C}_{27} \mathrm{H}_{19} \mathrm{~N}_{6} \mathrm{BrS}$ (539). Found: $\mathrm{C}, 59.89 ; \mathrm{H}, 3.45 ; \mathrm{N}$, 15.39; $\mathrm{Br}, 14.61 ; \mathrm{S}, 5.80 \%$.

\section{- 3-Aryl-5-methyl-2-(3-(pyridin-4-yl)-5H-[1,2,4]triazino[5,6-b]indol-5-yl) thiazolidin-4-ones (6a-c)}

A mixture of $4(0.01 \mathrm{~mol})$, thiolactic acid $(0.05 \mathrm{~mol})$ and sodium acetate anhydrous $(5 \mathrm{~g})$ in dry dioxane $(100 \mathrm{ml})$ was heated under reflux for $6 \mathrm{~h}$, cooled then poured onto ice and neutralized with $\mathrm{NaHCO}_{3}$. The solid produced filtered off and crystallized from EtOH to give compounds 6a-c.

6a: Yield 66\%, M.p: $210^{\circ} \mathrm{C}-212^{\circ} \mathrm{C}$. IR $(v) \mathrm{cm}^{-1}: 3163$ (aromatic CH), 2900, 2880 (aliphatic $\mathrm{CH}), 1718(\mathrm{C}=\mathrm{O}), 1616(\mathrm{C}=\mathrm{N}), 1463$ (aliphatic $\mathrm{CH}), 1234(\mathrm{C}-\mathrm{F})$, 1149 (C-S-C), 983, 846 (aromatic CH), 746 (C-F). ${ }^{1} \mathrm{HNMR}$ (DMSO- $d_{6}$ ) $\delta$ ppm: $11.43\left(s, 1 \mathrm{H}, \mathrm{OH}\right.$ of $\left(-\mathrm{CH}-\mathrm{C}=\mathrm{O} \rightleftharpoons-{ }^{\mathrm{C}}=\mathrm{C}-\mathrm{OH}\right), 8.95(s, 1 \mathrm{H}), 8.88,8.87(d, d, 2 \mathrm{H}$, pyridine), 7.81, 7.80 (each $d, d, 2 \mathrm{H}, \mathrm{C}-\mathrm{F}), 7.79-7.52(\mathrm{~m}, 2 \mathrm{H}$, of aryl), $7.42-7.40$ ( $2 \mathrm{H}$, pyridine), 7.39 - 6.98 ( $m, 4 \mathrm{H}$ of benzo protons), 3.37 ( $s, 1 \mathrm{H}, \mathrm{C} 5-\alpha \mathrm{H}$, thiazolidinone), $2.51(s, 3 \mathrm{H}, \mathrm{Me}) ;{ }^{13} \mathrm{CNMR}$ (DMSO- $\left.d_{6}\right) \delta$ ppm: $163.1(\mathrm{C}=\mathrm{O}), 150.85$ (C-S), $142.75(\mathrm{C}-\mathrm{F}), 139.23,135.78(\mathrm{C}=\mathrm{N}), 132.20(\mathrm{C}-\mathrm{F}), 129.45$ - 121.21 (aromatic carbons), 119.57, 111.33 (C5 - C6 of 1,2,4-triazine), $39.52\left(\mathrm{CH}_{3}\right)$.

Anal. Calcd. C, 60.26; H, 3.27; N, 18.34; F, 4.44; S, 6.98\% for $\mathrm{C}_{23} \mathrm{H}_{15} \mathrm{~N}_{6} \mathrm{FSO}_{2}$ (458). Found: C, 60.11; H, 3.02; N, 18.12; F, 4.35; S, 6.80\%.

6b: Yield 70\%, M.p: $228^{\circ} \mathrm{C}-230^{\circ} \mathrm{C}$. IR $(v) \mathrm{cm}^{-1}: 3080$ (aromatic CH), 2950, 2880 (aliphatic $\mathrm{CH}$ ), $1610(\mathrm{C}=\mathrm{N}), 1488,1444$ (deformation of $\mathrm{CH}$ ), 1085 (C-S-C), 910, 890, 815 (aromatic CH), 710, 700 (C-Cl). Anal. Calcd: C, 54.22; H, $2.75 ; \mathrm{N}, 16.50 ; \mathrm{Cl}_{2}, 13.94 ; \mathrm{S}, 6.28 \%$ for $\mathrm{C}_{23} \mathrm{H}_{14} \mathrm{~N}_{6} \mathrm{Cl}_{2} \mathrm{SO}_{2}$ (509). Found: $\mathrm{C}, 53.95 ; \mathrm{H}$, $2.55 ; \mathrm{N}, 16.18 ; \mathrm{Cl}, 13.85 ; \mathrm{S}, 6.01 \%$.

6c: Yield 58\%, M.p: $215^{\circ} \mathrm{C}-218^{\circ} \mathrm{C}$. IR (v) $\mathrm{cm}^{-1}: 3055$ (aromatic CH), 2900 (aliphatic $\mathrm{CH}), 1610(\mathrm{C}=\mathrm{N}), 1580(\mathrm{C}=\mathrm{N}), 1480$ (deformation $\mathrm{CH}), 1090$ (C-S-C), 910, 870, 820 (aromatic CH), 700 (C-Br). Anal. Calcd: C, 53.17; H, 2.89; N, $16.18 ; \mathrm{Br}, 15.41 ; \mathrm{S}, 6.16 \%$ for $\mathrm{C}_{23} \mathrm{H}_{15} \mathrm{~N}_{6} \mathrm{BrSO}_{2}$ (519). Found: $\mathrm{C}, 52.89 ; \mathrm{H}, 2.71 ; \mathrm{N}$, $15.95 ; \mathrm{Br}, 15.19 ; \mathrm{S}, 5.89 \%$.

\section{Conclusion}

A simple route to obtain various halogenated 4-thiazolidinones bearing a 1,2,4-triazino[5,6- $b$ ]indole moiety as fungicidal agents have been deduced starting from condensation of 5-formyl-1,2,4-triazinoindole with halogenated primary aromatic amines, followed by 1,2-addition of thiophenol and/or thiolactic acid. The fluorinated substituted showed a higher activity towards the tested fungi.

\section{Conflicts of Interest}

The author declares no conflicts of interest regarding the publication of this paper. 


\section{References}

[1] Gladych, J.M., Hornby, R., Hunt, J.H., Jack, D., Boyle, J.J., Ferlauto, R.J., Haff, R.F., Kormendy, C.G., Stanfield, F.J. and Stewart, R.C. (1972) Antiviral Agents. 5H-asTriazino[5,6-b]indoles. Journal of Medicinal Chemistry, 15, 277-281. https://doi.org/10.1021/jm00273a017

[2] Shaban, M.A.E., Nasr, A.Z. and Morgaan, A.E.A. (1999) Sterically Controlled Regiospecific Heterocyclization of 3-Hydrazino-5-Methyl-1,2,4-Triazino[5,6-b] Indole to 10-Methyl-1,2,4-Triazolo[4',3':2,3]1,2,4-triazino[5,6-b]indoles. Il Farmaco, 54, 800-809. https://doi.org/10.1016/S0014-827X(99)00107-X

[3] Kinsman, O.S., Livermore, D.G. and Smith, C. (1993) Antifungal Properties in a Novel Series of Triazino[5,6-b]indoles. Antimicrobial Agents, and Chemotherapy, 37, 1243-1246. https://doi.org/10.1128/AAC.37.6.1243

[4] Monge, A., Palop, J., Ramirez, C., Font, M. and Fernandez-Alvarez, E. (1991) New 5H-1,2,4-triazino[5,6- $b$ ]indole and Aminoindole Derivatives. Synthesis and Studies as Inhibitors of Blood Platelet Aggregation, Anti-Hypertensive Agents and Thromboxane Synthetase Inhibitors. Nouveaux dérivés $5 H$-1,2,4-triazino[5,6- $b]$ indole et de l'aminoindole. Synthèse et évaluation comme inhibiteurs de l'agrégation plaquettaire, agents anti-hypertenseurs et inhibiteurs de la thromboxane synthétase. European Journal of Medicinal Chemistry, 26, 179-188. https://doi.org/10.1016/0223-5234(91)90027-K

[5] Kgokong, J.L., Smith, P.P. and Matsabisa, G.M. (2005) 1,2,4-Triazino-[5,6b]indole derivatives: Effects of the Trifluoromethyl Group on in Vitro Antimalarial Activity. Bioorganic \& Medicinal Chemistry, 13, 2935-2942. https://doi.org/10.1016/j.bmc.2005.02.017

[6] Shelke, S.M. and Bhosale, S.H. (2005) Synthesis, Antidepressant Evaluation and QSAR Studies of Novel 2-(5H-[1,2,4]triazino[5,6-b]indol-3-ylthio)-N-(substituted phenyl)acetamides. Bioorganic \& Medicinal Chemistry Letters, 20, 4661-4664.

https://doi.org/10.1016/j.bmcl.2010.05.100

[7] Sharma, R., Pandey, A.K., Shivahare, R., Srivastava, K., Gupta, S. and Chauhan, P.M. (2014) Triazino Indole-Quinoline Hybrid: A Novel Approach to Antileishmanial Agents. Bioorganic \& Medicinal Chemistry Letters, 24, 298-301.

https://doi.org/10.1016/j.bmcl.2013.11.018

[8] Tomchin, A.B., Uryupov, O.Y., Zhukova, T.I., Kuznetsova, T.A., Kostycheva, M.V. and Smirnov, A.V. (1997) Thiourea and Thiosemicarbazide Derivatives: Structure, Transformations, and Pharmacological Activity. Part II. Antihypoxic Activity of 1,2,4-triazino[5,6-b]indole Derivatives. Pharmaceutical Chemistry Journal, 31, 125133. https://doi.org/10.1007/BF02464663

[9] Tomchin, A.B., Uryupov, O.Y. and Smirnov, A.V. (1997) Thiourea and Thiosemicarbazide Derivatives: Structure, Transformations, and Pharmacological Activity. Part III. Antihypoxic and Antiinflammatory Activity of 1,2,4-triazino[6,5-b]indole Derivatives. Pharmaceutical Chemistry Journal, 31, 632-637.

https://doi.org/10.1007/BF02464248

[10] Abdel-Rahman, R.M., Seada, M., Fawzy, M.M. and El-Baz, I. (1993) Synthesis and Anti-Canceranti Human Immune Virus Activities of Some New Thioether Bearing a 1,2,4-Triazine-3-Hydrazones. Farmaco, 48, 397-406.

[11] Abdel-Rahman, R.M. (2001) Chemoselective Heterocyclization and Pharmacological Activities of New Heterocycles a Review Part v-Synthesis of Biocidal 4-Thiazolidinones Derivatives. BollettinoChimicofarmaceutico, 140, 401-410.

[12] Abdel-Rahman, R.M. and Ali, T.E. (2013) Synthesis and Biological Evaluation of 
Some New Polyfluorinated 4-Thiazolidinone and $\alpha$-Aminophosphonic Acid Derivatives. Monatsheftefür Chemie-Chemical Monthly, 144, 1243-1252.

https://doi.org/10.1007/s00706-013-0934-6

[13] Makki, M.S.T., Alfooty, K.O., Abdel-Rahman, R.M. and El-Shahawi, M.S. (2016) Synthesis, Voltammetric and Analytical Applications of Some Fluorine Substituted Spirosteroidalthiazolidin-4-One Derivatives of Sulfa Drugs. Journal of the Chinese Chemical Society, 63, 189-198. https://doi.org/10.1002/jccs.201500240

[14] Gould, J.C. (1952) The Determination of Bacterial Sensitivity to Antibiotics. Edinburgh Medical Journal, 59, 178.

[15] Singh, A., Dhakarey, R. and Saxena, G.C. (1996) Magnetic and Spectral Behavior of Semicarbazone Derivatives of Manganese(Ii), Copper(Ii), Iron(Iii) and Chromium (Iii) and Their Antimicrobial Screening. Journal of the Indian Chemical Society, 73, 339-342. 